\title{
Advanced Brushless DC Motor Drive without Position Sensor for Home Appliances
}

\author{
Dae-kyong Kim *, Ji-Young So * and Dong-Hwa Jung**
}

\begin{abstract}
This paper describes advanced brushless DC motor drive without position sensor for home appliances with compressor to reduce the pulsating currents and vibration. The proposed method limits the motor currents during starting period and reduces commutation torque ripple during sensorless operating period. Experimental results show that the proposed method implemented in an inverter for a BLDC motor driven compressor considerably reduces not only the pulsating currents but also vibration of the home appliances.
\end{abstract}

Keywords: BLDC, Compressor, Sensorless, ZCP

\section{Introduction}

During the last decades, energy saving has been one of the important issues in home appliances [1]. Compressor is the device which consumes most of electrical energy in airconditioner and refrigerator. Therefore, a lot of efforts have been made to improve the energy efficiency of the compressor. For these reasons, the BLDC motor is expanding its application in compressor markets. The rotor position sensing devices can not be used in the BLDC motor driven compressor. Because of environment of high temperature and pressure inside, the compressor can cause permanent damage. Thus, sensorless controlled BLDC motors have been used for driving compressors [2-6].

Among various sensorless control strategies for the BLDC motor, the sensorless control method based on the detection of zero crossing point (ZCP) of back-EMF has been widely used for low cost applications because of its simplicity. However, the ZCP of the back-EMF can not be obtained when the BLDC motor is at standstill or operating at nearly zero speed. In addition, the commutation current ripple is generated in the course of nature in BLDC drive. Therefore, not only a special starting control is required for smooth starting and reliable transfer to sensorless control, but also commutation current ripple reduction method without current sensor is required for reducing torque ripple in a sensorless control mode [4-6].

This paper describes an advanced sensorless BLDC

\footnotetext{
* Dept. of Electrical Control Engineering, Sunchon National University,

Korea. (dkkim@sunchon.ac.kr,drsojy@sunchon.ac.kr)

**Corresponding Author: Dept. of Electrical Control Engineering, Sunchon

National University, Korea. (hwa777@sunchon.ac.kr)

Received17 January 2013; Accepted 08 February 2013
}

motor drive which can be a good solution for low cost and high performance in home appliances. The proposed method limits the peak currents during starting period by doing commutation according to the level of measured phase currents without current control loop and reduces commutation current ripple by directly measuring commutation interval from motor terminal voltage waveforms.

The proposed solution is implemented on commercial refrigerator using a BLDC motor driven reciprocating compressor. Experimental results show that the proposed method considerably reduces not only instantaneous peak currents of the motor but also vibrations of the compressor compared to the conventional method.

\section{Sensorless Control of BLDC Motor [2]}

The back-EMF's $e_{a}, e_{b}$ and $e_{c}$ of BLDC motor have trapezoidal shapes and the currents $i_{a}, i_{b}$ and $i_{c}$ needed to produce a steady state torque without torque pulsations are shown in fig. 1.

In a position sensorless BLDC motor drive, commutation points of the inverter can be obtained by knowing the zerocross-point (ZCP) of the back-EMF and a speed dependent period of time delay [2]. The phase back-EMF induced in the stator windings of a BLDC motor is trapezoidal so that the ZCP of the back-EMF can be detected by monitoring the terminal voltage waveform of a silent phase. The instance when the terminal voltage of the silent phase match with the half DC link voltage, during which point switching devices are turned on, is the zero crossing point of the back-EMF. The commutation points are estimated like this: 


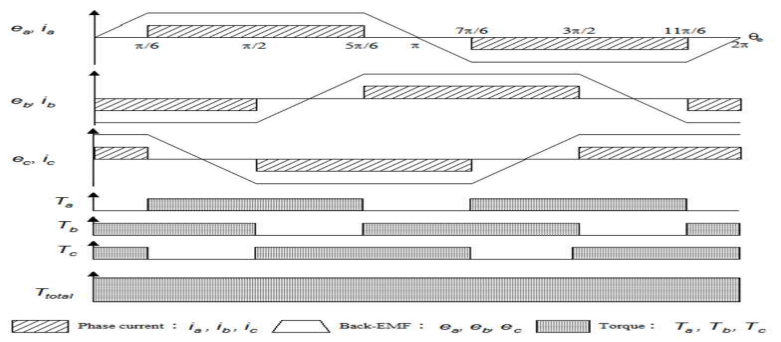

Fig.1. Current, back-EMF and torque waveforms of BLDC motor

$$
\begin{gathered}
T_{c m t}(k)=T_{z c p}(k)+\frac{1}{2} \Delta T_{Z C P}(k) \\
\Delta T_{Z C P}(k)=T_{Z C P}(k)-T_{Z C P}(k-1)
\end{gathered}
$$

where, $\operatorname{Tcmt}(\mathrm{k})$ is the commutation time and $\operatorname{TZCP}(\mathrm{k})$ is the zero crossing time of the back-EMF.

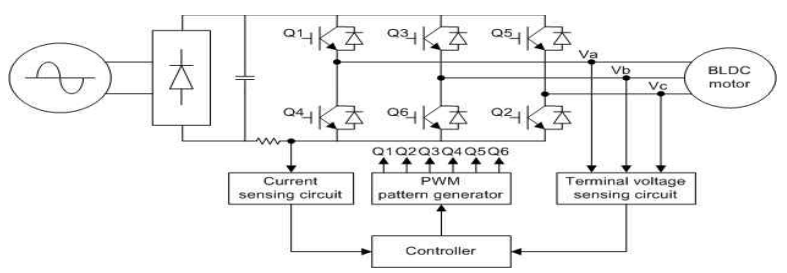

(a)

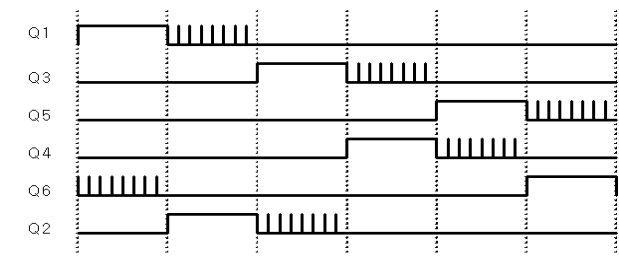

(b)

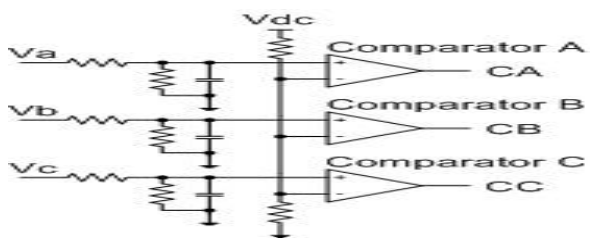

(c)

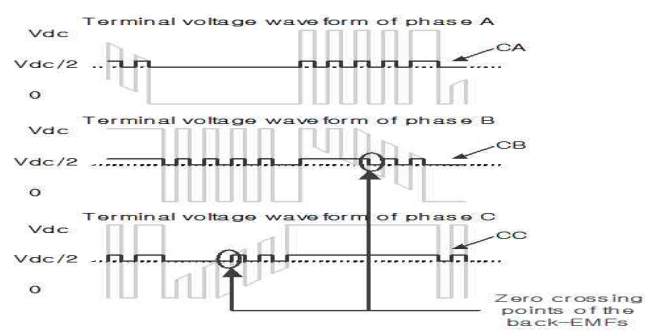

(d)

Fig.2. (a) configuration of a BLDC motor drive, (b) switching pattern, (c) terminal voltage sensing circuit and (d) terminal voltage waveforms
Fig. 2(a) depicts the configuration of a position sensorless BLDC motor drive, fig. 2(b) the switching pattern of the outgoing phase unipolar PWM, fig. 2(c) the terminal voltage sensing circuit, and fig. 2(d) the motor terminal voltage waveforms when the out-going phase unipolar PWM is applied. The terminal voltage sensing circuit in fig. 2(c) compares the terminal voltage of each phase with the half DC link voltage. In fig. 2(c), capacitor $\mathrm{C}$ is used to reduce switching noises included in the terminal voltage.

The sensorless control method based on terminal voltage sensing cannot be used during low speeds since the back-EMF is zero at rest and proportional to the speed.

\section{Starting Method}

In the conventional 'align and go' starting method of the BLDC motor driven compressor, the BLDC motor is commutated according to the predetermined pattern regardless of the real rotor position during open loop acceleration mode [2-3]. During the open loop acceleration mode, current waveforms are affected by commutation point. Fig. 3 shows the relation between current waveform and commutation point when the voltage applied to the BLDC motor is constant. If the commutation point lags behind proper commutation point, the phase current before commutation goes up since the backEMF of the corresponding phase after exact commutation point is falling down. On the other hand, the phase current after commutation goes up in the case of leaded commutation. By using these phenomena, the large instantaneous peak current, caused by improper commutation point in the conventional starting method, during open loop acceleration mode can be reduced.

When the acceleration speed is set to slightly slower than the mechanical response speed of the reciprocating compressor, the commutation points lag behind the correct commutation points. In this case, the phase current waveform is shown as fig. 4. The average current value $(\mathrm{TH})$ is obtained by averaging non-commutated phase currents after transient period of commutation.

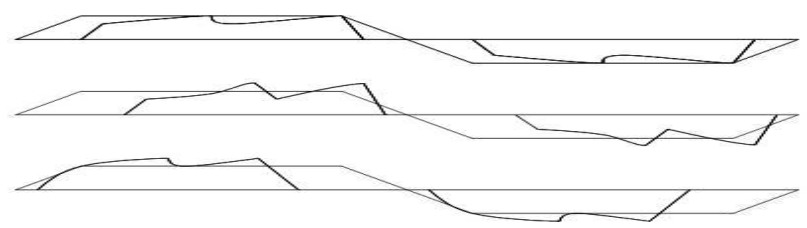

Fig. 3. Current waveforms as the relation of the rotor position and the commutation point (upper: proper commutation, middle: lagged commutation, lower: leaded commutation) 
The over current value (TH over) is set to the $110 \sim 120 \%$ of $\mathrm{TH}$ and $\delta \mathrm{t}$ is the time of measured TH_over. If the phase current goes over $\mathrm{TH}$ _over then it can be assumed that the correct commutation point is elapsed. The proposed method commutates the BLDC motor during open loop acceleration mode depending on the level of the phase current. On the other hand, the conventional starting method commutates the BLDC motor according to the predetermined commutation period. Therefore, the proposed starting method can considerably reduce the error of commutation point compared to the conventional starting method.

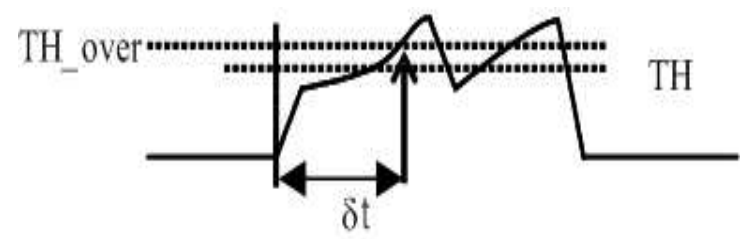

Fig. 4. Relation of average current $(\mathrm{TH})$ and over current value (TH_over)

Fig. 5 shows the flowchart of the proposed starting method. First, any two phases of the stator are energized to align the rotor and then the phase commutation is carried out. Second, the next phase commutation time ( $T_{-}$com) is calculated and then the internal Timer is reset to zero. Third, the average current $(\mathrm{TH})$ is obtained from the shunt resistor of the IPM and TH_over is calculated from TH. Fourth, the phase current detected from the shunt resistor is compared with $\mathrm{TH}$ _over. If the current is higher than $\mathrm{TH}$ _over, the phase commutation is carried out. On the other hand, if the current is not over TH_over, the current detection and comparison action continue to do until the internal timer reaches $\mathrm{T}$ _com. If the internal Timer increases to T_com, the phase commutation is carried out. Finally, if the synchronous acceleration frequency is reached to the set frequency, the synchronous acceleration mode is transferred to the sensorless control mode.

\section{Commutation Current Ripple Reduction Method}

\subsection{Analysis of commutation current ripple}

Average voltage $\mathrm{V}_{\mathrm{m} 1}$ applied to a non-commutated phase before commutation is

$$
V_{m 1}=\frac{V_{d c}}{2} D_{a}
$$

where, $\mathrm{V}_{\mathrm{dc}}$ is the inverter dc-link voltage, $\mathrm{D}_{\mathrm{a}}$ is PWM duty ratio during two phase conduction period.Fig. 6 shows current paths in out-going phase unipolar PWM scheme when the phase current is being transferred from phase A to phase B.

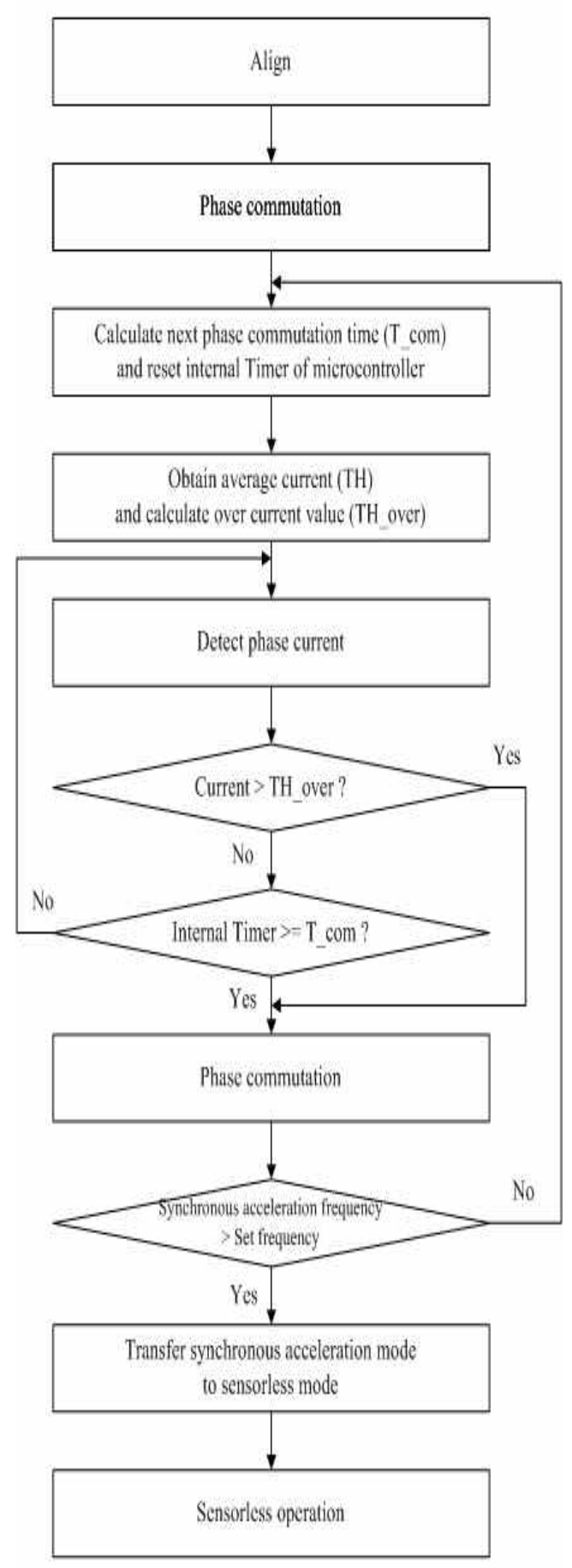

Fig. 5. Flowchart of the proposed starting method 

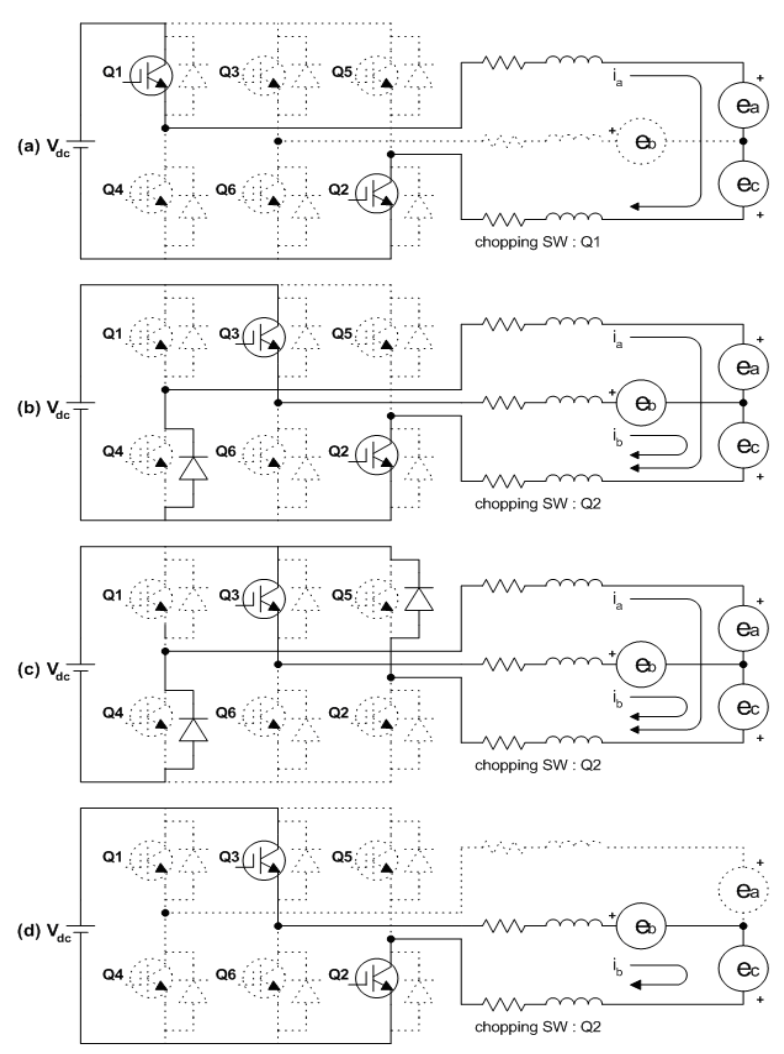

Fig. 6. Equivalent circuit during phase current commutation (a) before commutation (b)(c) during commutation (d) after commutation

From fig. 6, the phase voltage equation during commutation is given as (4)

$$
\begin{aligned}
& V_{a}^{\prime}=0=R \cdot i_{a}+L \cdot \frac{d i_{a}}{d t}+e_{a}+V_{n} \\
& V_{b}^{\prime}=V_{s}=R \cdot i_{b}+L \cdot \frac{d i_{b}}{d t}+e_{b}+V_{n} \\
& V_{c}^{\prime}=V_{s}(1-S)=R \cdot i_{c}+L \cdot \frac{d i_{c}}{d t}+e_{c}+V_{n} \\
& V_{n}=\frac{V_{s}}{3}(2-S)-\frac{e_{a}+e_{b}+e_{c}}{3}
\end{aligned}
$$

In case of an out-going phase unipolar PWM, average voltage $\mathrm{Vm} 2$ applied to a non-commutated phase is

$$
V_{m 2}=\frac{\mathrm{V}_{\mathrm{dc}}\left(2 D_{b}-1\right)}{3}-\frac{e_{a}+e_{b}+e_{c}}{3}
$$

where $\mathrm{D}_{\mathrm{b}}$ is $\mathrm{PWM}$ duty ratio during commutation.

From equation (3) and (8), it is apparent that the average voltage of the non-commutationed phase is disturbed by commutation. This voltage disturbance generates pulsating current. Since generated torque during commutation is proportional to the non-commutated phase current, the pulsating current causes undesirable torque ripple during the commutation.

\subsection{Voltage disturbance rejection method}

In order to minimize the pulsating current, the PWM duty ratio during commutation must be modified as equation (9) in order that the average voltage of the non-commutated phase maintains constant value, that is, $\mathrm{Vm} 2=\mathrm{Vm}$.

$$
D_{b}=\frac{1}{2}+\frac{3}{4} D_{a}+\frac{e_{a}+e_{b}+e_{c}}{2 V_{d c}}
$$

If the phase back-EMF assumes constant in the commutation period and rotational velocity of motor, the phase back-EMF is given as follows.

$$
e_{a}=e_{b}=-e_{c}=K_{e} \omega_{m}
$$

Equation (9) is adjusted as in equation (11)

$$
D_{b}=\frac{1}{2}+\frac{3}{4} D_{a}+\frac{K_{e} \omega_{m}}{2 V_{d c}}
$$

where Ke is back-EMF constant, and $\omega_{\mathrm{m}}$ is mechanical speed of a motor.

If the compensation input to reject voltage disturbance is still applied to $\mathrm{Db}$ after the commutation, current spikes will be generated due to over-compensation. In order to apply the strategy for reducing commutation torque ripple to a BLDC motor drive, the accurate start and end point of commutation must be known.

For prohibiting the over-compensation, the compensation input $\mathrm{Db}$ is adjusted from the commutation interval. As the measured commutation interval is $t_{c}$, the compensation input Db' is adjusted like equation (12)

$$
D_{b}^{\prime}=D_{b} \times \frac{t_{c}}{T}
$$


where $\mathrm{T}$ is PWM period. The adjusted input $\mathrm{D}_{\mathrm{b}}$ is applied to an inverter only during the next commutation.

\subsection{Measurement of commutation interval}

When the voltage disturbance rejection method is applied to the system, the duration of commutation must be known. In order to know the duration of commutation, a current sensor has been used. However, this paper introduces the novel method to measure the commutation interval without current sensors.

As explained in preview section, the position sensorless controller already uses the terminal voltages for obtaining the ZCP of phase back-EMF. If the terminal voltages can give information related to the commutation interval for the voltage disturbance rejection method, the current sensors are no more required. When using the out-going phase unipolar PWM scheme, the terminal voltage of a silent phase remains positive (or zero) DC bus voltage during commutation and is lower (or higher) than half the DC bus voltage until the back EMF of the silent phase reaches zero. By measuring the transition time of the comparator output after starting the commutation, hence, the duration of commutation can be known as shown in Fig. 3.

\subsection{Implementation of the proposed strategy}

Fig. 7 shows the configuration of the proposed controller. In Fig 7, the speed controller determines the PWM duty ratio, $\mathrm{u}$, during two-phase conduction period. When the commutation starts, a PWM duty ratio, $\mathrm{u} 1$, is changed to $\mathrm{u} 2$. The commutation interval detector outputs two kinds of control signals, $\mathrm{G}_{-}$con and $\mathrm{U}$ _con. $\mathrm{G}$ _con adjusts gain $\mathrm{K}$ and $\mathrm{U}$ _con changes $\mathrm{u} 2$ to $\mathrm{u} 1$ after the end of commutation period and gives the starting point of PWM carrier signal to synchronization of gating signal. u3 goes to PWM pattern generator.

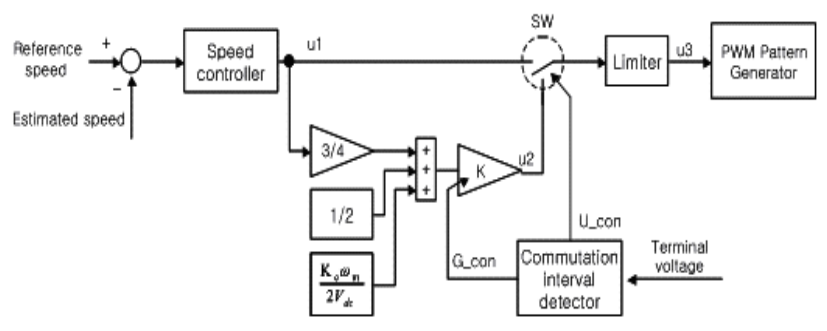

Fig. 7. Configuration of the proposed controller

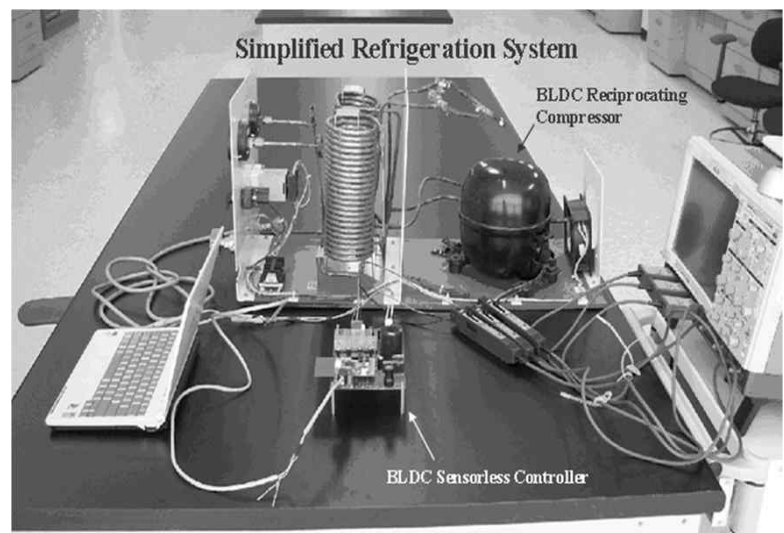

Fig. 8. Experimental test-bed

Fig. 8 shows the experimental test-bed for the simplified refrigeration system. In this experiment, a low-cost fixed-point digital signal processor (DSP), TMS320LF2406A, has been used for implementation of the proposed starting method.

\subsection{Experimental Results}

Table 1 shows the electrical parameters of the used BLDC motor to examine the performance of the proposed starting method.

Table 1.Motor parameters

\begin{tabular}{|c|c|}
\hline Rated power & $200[\mathrm{w}]$ \\
\hline Number of pole & 4 \\
\hline Line to line resistance & $7.5[\Omega]$ \\
\hline Line to line inductance & $0.021[\mathrm{H}]$ \\
\hline Back-EMF constant & $57.78\left[\mathrm{~V}_{\mathrm{rms}} / \mathrm{krpm}\right]$ \\
\hline
\end{tabular}

When the commutation interval has the periodic shapes, the look-up table can be used for keeping the commutation interval.

Fig. 9 (a) and (b) show starting current waveforms and commutation signals of the conventional method and the proposed method when the pressure difference between the suction and the discharge port of the compressor is 0 [kgf/cm2], respectively. The lower one of each fig. 9 (a) and (b) show the enlarged current waveforms and commutation signals. In the conventional method, the commutation points are pre-determined so that the commutation signals in fig. 9 (a) show fixed pattern. The proposed method, on the other hand, adjusts the commutation point depending on the level of phase current so that the commutation signals of the proposed method show irregular pattern. 


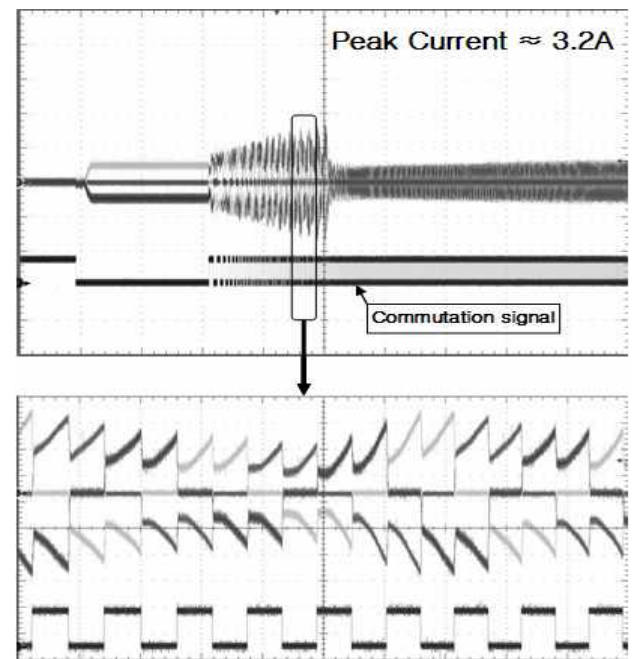

(a)

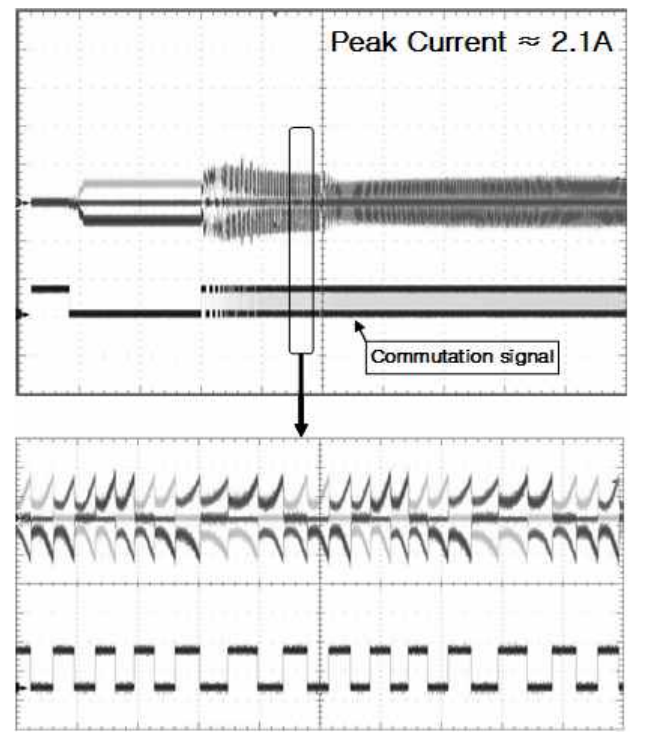

(b)

Fig. 9. Starting current waveforms at $0 \mathrm{kgf} / \mathrm{cm}^{2}$ pressure difference between suction and discharge port of the compressor (a) conventional method (b) proposed method

Fig. 10 (a) and (b) show current waveforms of the [kgf/cm2] pressure differences between the suction and the discharge port of the compressor, respectively. It is noted that the proposed starting method remarkably reduces the instantaneous peak currents during starting mode.

The experimental results have proved that the proposed method can reduce instantaneous peak current considerably at not only start-up mode but also sensorless mode. In addition, since phase current is strongly related to torque and vibration [5-6], the proposed method is very effective at the vibration suppression of the reciprocating compressor.

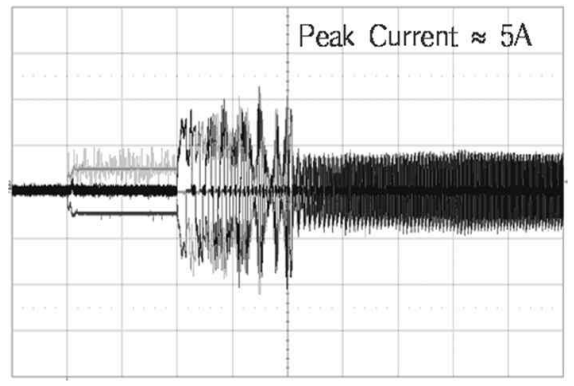

(a)

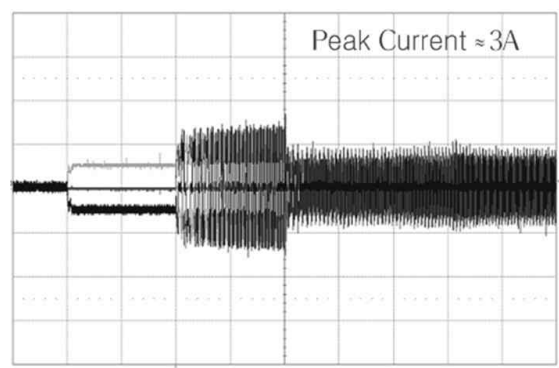

(b)

Fig.10. Starting current waveforms at $3.0 \mathrm{kgf} / \mathrm{cm}^{2}$ pressure difference between suction and discharge port of the compressor (a) conventional method (b) proposed method

Fig. 11 and 12 show the experimental result of phase current at $1800 \mathrm{rpm}$ and $2000 \mathrm{rpm}$, respectively. Figs. 11(a) and 12(a) show the experimental results without the proposed method. The phase current spikes are generated during the commutation. Figs. 11(b) and 12(b) show that the proposed method remarkably reduces the current spikes. Conventional method and the proposed method at 3

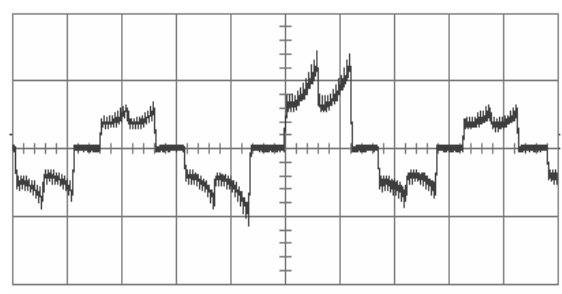

(a)

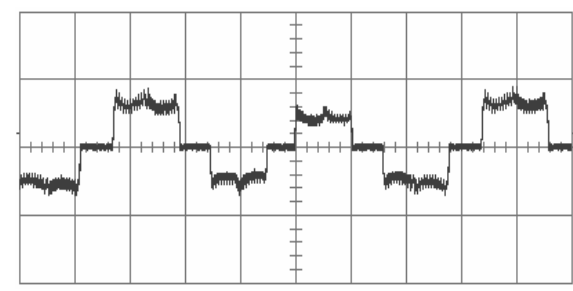

(b)

Fig.11. Phase currents at $1800 \mathrm{rpm}$ (a) conventional method (b) proposed method (2A/div., 5msec/div.) 
(a)

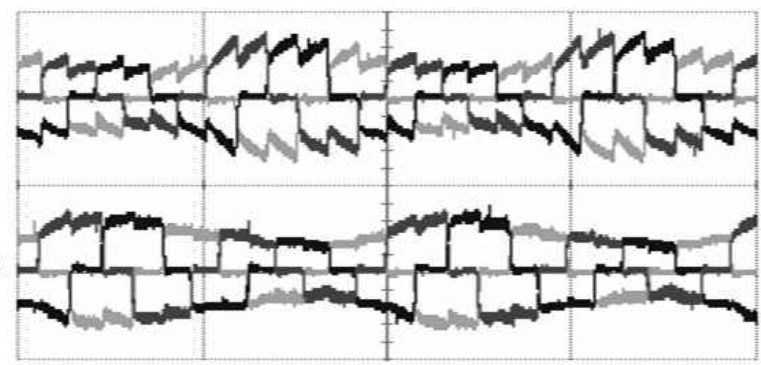

Fig.12. Phase currents at $2000 \mathrm{rpm}$ (a) conventional method (b) proposed method

\section{Conclusion}

This paper proposed advanced brushless DC motor drive without position sensor for home appliances. A summary of the work and some conclusion are as follows.

- The experimental results showed that the proposed method considerably reduces not only instantaneous peak currents but also vibration of the reciprocating compressor.

- The proposed method makes possible low-cost home appliances using BLDC motor, such as refrigerator and air-conditioner, to obtain the good performance.

\section{Acknowledgements}

This work was supported by the New \& Renewable Energy of the Korea Institute of Energy Technology Evaluation and Planning (KETEP) grant funded by Ministry of Knowledge Economy, Korea. (No. 20113030060010)

\section{References}

[1] Electric Power Research Institute, "Electric Motors; Markets, Trends, and Application," Tech. Rep. TR-100423, Jun. 1992.

[2] K. Iizuka, et al., "Microcomputer Control for Sensorless Brushless Motor," IEEE Trans. on Industry Applications, vol. 21, no. 3, pp. 595-601, May 1985.

[3] P. Pillay and R. Krishnan, "Modeling, Simulation and Analysis of Permanent-Magnet Motor Drives, Part II: The PermanentMagnet Synchronous Drive,” IEEE Trans. Ind. Appl., vol. IA-25, No. 2, pp. 265-273, Mar./Apr. 1989.

[4] Kwan-yuhl Cho, "Sensorless Control for a PM Synchronous Motor in a Single Piston Rotary Compressor," Journal of Power Electronics, Vol. 6, No. 1, pp. 29-37, Jan. 2006

[5] Dae-kyong Kim, Kwang-woon Lee, Byung-il Kwon, "Commutation Torque Ripple Reduction in a Position Sensorless Brushless DC Motor Drive," IEEE Trans. On Power Electronics, vol. 21, No. 6, pp. 1762-1768, Nov. 2006
[6] Kwang-woon Lee, Dae-kyong Kim, Byung-Taek Kim, Byung-il Kwon, "A Novel Starting Method of the SPM-type BLDC Motors without Position Sensor for Reciprocating Compressor", IEEE Transations on Industry Applications, Vol. 44, No. 1, January/February 2008

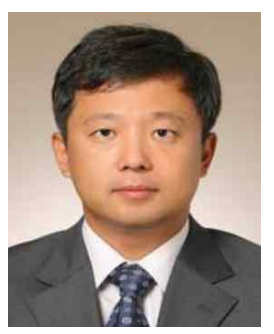

Dae-kyong Kim received his M.S. degree in Electrical Engineering and his Ph.D. degree in the Department of Electronic, Electrical, Control and Instrumentation Engineering from Hanyang University, Korea in 2001 and 2007, respectively. From 2001 to 2005, he was a Senior Engineer in the Home Appliances R\&D Center where he was engaged in research and development of sensorless motor drive systems for refrigerator and air-conditioner at the Samsung Electronics, Korea. From 2005 to 2011, he was a Center Director of Digital Convergence Center at the Korea Electronics Technology Institute (KETI). Since 2011, he has been with the Department of Electrical Control Engineering, Sunchon National University, Korea as an assistant professor. His current research is in analysis, design, and drives of electrical machines for energy saving and high-performance. He received several Best Paper Awards from the Korea Institute of Electrical Engineers, ICEMS2007 and IEEE INTELEC2009. He received a R\&D award from Samsung Electronics, a staff award from Korea Electronics Technology Institute and a mayor award from Gwangju-city, Korea. He served as Cochairs and secretary for IEEE INTELEC2009, ICEMS2010, IEEE VPPC2012, and CEFC2012, and other domestic and international conference.

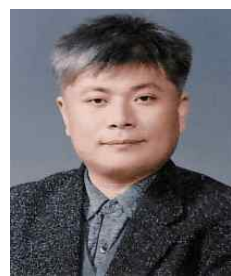

Ji-Young Soo received the B.S, M.S and $\mathrm{Ph} . \mathrm{D}$. degrees in electrical engineering from Yonsei University, Seoul, Korea, in 1983, 1985, and 1990 respectively. Since 1990, he has been with the department of Electrical and control Engineering, Sunchon National University, Korea, where he is currently a Professor. From 1998 to 1999, he was a Visiting Scholar with UNSW, Sydney, Australia. His fields of interest include electric materials, magnetic materials, and microcontroller based smart controller. 


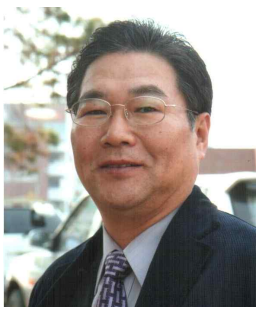

Dong-hwa Chung received his Ph.D. degree in Electric Engineering from Hanyang University, Korea, in 1987. He is a professor of Electrical Control Engineering at Sunchon National University, Korea. His research interests include fuzzy control, neural networks, motor control and photovoltaic power. His current research focuses on maximum power point tracking control of PV generation. He has received about twenty awards such as the best paper award and academy research award etc, from the Korean Institute of Electrical Engineers and the Korean Institute of Illuminating and Electrical Installation Engineers etc,. He has authored about 100 papers since 1983. His research interests include power electronics, electric motor control, artificial intelligent control and photovoltaic generation. 